\title{
Non breathing-related sleep fragmentation and imaging markers in patients with atherosclerotic cerebral small vessel disease (CSVD), a cross- sectional case-control study
}

\author{
Jihui Wang \\ Sun Yat-Sen University \\ Xiaodong Chen \\ Sun Yat-Sen University \\ Jinchi Liao \\ Sun Yat-Sen University \\ Li Zhou \\ Guangdong Pharmaceutical University \\ Hongying Han \\ Sun Yat-Sen University \\ Jiong Tao \\ Sun Yat-Sen University \\ Zhengqi Lu (D lzq1828@outlook.com)
}

\section{Research article}

Keywords: Cerebral vascular disease®Sleep囚Polysomnography, Fragmentation, Markers

Posted Date: October 11th, 2019

DOI: https://doi.org/10.21203/rs.2.15917/v1

License: (c) (i) This work is licensed under a Creative Commons Attribution 4.0 International License.

Read Full License 


\section{Abstract}

Objective Evidence of a relationship between non breathing-related sleep fragmentation and imaging markers of the brain in cerebral small vessel disease (CSVD) patients is scarce. The purpose of the present study was to investigate the relationship in CSVD patients living in China, where CSVD is a major pathogenesis underlying stroke.

Methods A group of 84 CSVD patients were prospectively recruited along with 24 age and sex matched normal controls. 3.0 T superconducting magnetic resonance imaging and overnight polysomnography were conducted in all the participants. Polysomnography parameters including sleep onset latency, total sleep time; sleep efficiency, wake after sleep onset, percentage of each sleep stage (N1, N2, N3 and REM) and arousal index were compared between the CSVD patients and normal controls, and the relationship between arousal index and CSVD markers was explored in the CSVD group.

Results Polysomnography measures showed that CSVD patients had significantly higher arousal index and wake after sleep onset, lower sleep efficiency and N-3 ratio compared to normal controls $(p<0.05)$. The results of ordinal logistic regression showed that higher arousal index was positively associated with the severity of periventricular white matter hyperintensity (OR 1.177, 95\% $\mathrm{Cl} 0.170$ to 2.295 ) and perivascular space (OR $1.245,95 \% \mathrm{Cl} 0.485$ to 2.124 ) in CSVD patients, after adjusting for all the independent variables.

Conclusions These preliminary results indicate that non breathing-related sleep fragmentation is common and related to the pathological markers of CSVD patients. Future prospective research is invited to establish the causal relationship between sleep parameters and CSVD pathology.

\section{Introduction}

The term cerebral small vessel disease (CSVD) refers to a series of clinical, imaging and pathological syndromes affecting small arteries, arterioles, capillaries and venules in the brain. The most common form is arteriolosclerosis which is also known as age-related and traditional vascular risk-factor-related small vessel disease[1].

Sleep fragmentation, characterized by recurrent and brief arousals that occur during nocturnal sleep, often affects the elderly and could lead to the destruction of sleep continuity and sleep quality [2]. The common cause of sleep fragmentation is obstructive sleep apnea syndrome(OSAS) which is regarded to be one of the non-traditional risk factors for cardio-cerebrovascular diseases and has been well concerned $[3,4]$. However, OSAS is relatively uncommon compared to non-breathing related sleep complaints such as insomnia [5]. The overall prevalence of insomnia symptoms ranges from $30 \%$ to $48 \%$ in the elderly and the prevalence of insomnia disorder ranges from $12 \%$ to $20 \%$ [6]. As far as we know, there are limited data evaluating the status of non-breathing-related sleep fragmentation in CSVD patients and its relationship with the pathological changes of the brain. Berezuk C (2015) assessed 26 cases of ischemic cerebrovascular disease using polysomnography(PSG) and magnetic resonance imaging $₫$ MRI $\bowtie$ and found 
that sleep efficiency(SE), wake after sleep onset(WASO) and N3 sleep duration were correlated with perivascular space(PVS) in basal ganglia. However, patients with OSAS were not excluded in this study which could cause confounding factors. The sample size of this study was relatively small and there was no control group which could reduce the significance of the study [7]. Other studies with larger sample have found that poor sleep quality [8] or long sleep duration [9] were related to white matter hyperintensity (WMH) in the brain, but the participants recruited were the elderly in the community or stroke-free urban sample, not the patients, and the sleep quality was evaluated only by subjective scales. Our previous study showed that sleep fragmentation was positively associated with cognitive impairment in CSVD patients, but we did not explore the underlying mechanisms, especially the relationship between sleep fragmentation and brain pathological changes. Moreover, the characteristics of sleep fragmentation could not be well understood due to the lack of healthy controls in this study [10].

In the present study, we explored the relationship between objective non breathing-related sleep parameters and neuroimaging markers in CSVD patients with a relatively larger sample and a group of normal control. We hypothesized that non breathing-related sleep fragmentation is common in CSVD patients and is positively associated with specific CSVD imaging markers.

\section{Methods}

Design [This was a cross-sectional case-control study in a single-center. The CSVD patients were consecutively recruited from outpatient clinics of the Third Affiliated Hospital of Sun Yat-sen University from September 2017 to December 2018. The healthy control group was the healthy spouse or sibling of the CSVD patients.

\section{Participants}

A total group of 84 CSVD patients were recruited following the inclusion/exclusion criteria, along with 24 normal controls matched with sex and age. All participants underwent structured interviews and physical examination by a study physician who confirmed the diagnosis.

\section{Inclusion criteria:}

1. Age $50-70 \mathrm{y}$.

2. Had at least one of the following vascular risk factors: hypertension, atherosclerosis, diabetes mellitus, past or current smoking.

3. Had the following symptoms of CSVD: lacunar syndromes, cognitive, motor (gait), dysphagia, or mood disturbances. Subjects who were eligible because of a lacunar syndrome were included only $>3$ months after the event to avoid acute effects on the outcomes.

4. Presenting with evidence of one or more MR markers of CSVD [11]: lacunars, WMHs with Fazekas grade 2(early confluent) or higher [12], visible PVSs and cerebral microbleeds (CMBs).

5. Controls without vascular risk factors, CSVD symptoms and MR marks of CSVD. 
6. Signed the written informed consent form.

\section{Exclusion criteria}

1. With larger subcortical or cerebral Water-shed infarctions $(>1.5 \mathrm{~cm})$ on MR as these are often embolic.

2. With large artery disease-carotid, vertebral or intracranial stenosis $>50 \%$;

3. With severe mental disorders such as schizophrenia, bipolar disorder or major depression, alcohol and/or other drug abuse or dependence;

4. With uncontrolled somatic disorders or sleep-disruptive comorbid medical condition (e.g., moderate to severe rheumatoid arthritis); needed immediate psychiatric (e.g., imminently suicidal patients) or medical care (e.g., patients with acute cardiac symptoms)

5. With breathing-related sleep disorders, restless legs syndrome, or circadian rhythm sleep disorder according to the criteria of the Diagnostic and Statistical Manual of Mental Disorders, Fifth Edition [13].

6. Apnea-hypopnea index $\geq 15$ or/and periodic limb movement-related arousal index $\geq 15$ per hour of sleep during a screening laboratory polysomnogram;

7. Patients with CSVD caused by other reasons such as hereditary, inflammatory and amyloidosis.

8. Being treated with medications that affect sleep within two weeks of initial screening.

9. Any inability to fulfill study data collection.

\section{Brain MRI scan:}

The General Electric 3 T superconducting MR scanner with 8 channels, head and neck coils was used for brain scanning. The acquisition sequences included 3D TOF-MRA, MRI T1Flair, T2WI, T2Flair and susceptibility weighted imaging (SWI). Images were rated by a certified and registered neuroradiologist in a blinded model for the presence of four CSVD makers as shown in the followings; the presence of each SVD makers was summed in an ordinal "SVD burden score" (range 0-4) [14].

Lacunes: rounded or ovoid lesions, $>3$ - and $<15-\mathrm{mm}$ diameter, in the basal ganglia, internal capsule, centrum semiovale, or brainstem, of CSF signal intensity on T2 and FLAIR, generally with a hyperintense rim on FLAIR, consistent with a previous acute small subcortical infarct or haemorrhage in the territory of one perforating arteriole. Presence of lacunes was defined as the presence of one or more lacunes (1 point if present).

WMHs: signal abnormality of variable size in the white matter that shows the following characteristics: hyperintensity on T2-weighted images such as fluid-attenuated inversion recovery, without cavitation (signal different from CSF). The severity of periventricular WMH (PWMH) and deep WMH (DWMH) were both coded according to the Fazekas scale from 0 to 3. Presence of WMH was defined as either (early) confluent DWMH (Fazekas score 2 or 3 ) or irregular PWMH extending into the deep white matter (Fazekas score 3) (1 point if present) [12]. 
PVSs: small ( $<3 \mathrm{~mm}$ ) punctate (if perpendicular) and linear (if longitudinal to the plane of scan) hyperintensities on T2 images in the basal ganglia, and the severity of PVS was rated on a validated semiquantitative scale from 0 to $4(0=$ no PVS, $1=<10 \mathrm{PVS}, 2=11$ to $20 \mathrm{PVS}, 3=21$ to $40 \mathrm{PVS}$, and $4=$ $>40$ PVS). The numbers refer to PVSs on one side of the brain; the higher score was used if there was asymmetry between the two sides. Presence of PVSs was counted if there were moderate to severe (grade 2-4) PVSs in the basal ganglia (1 point if present) [15].

CMBs: small (generally 2-5 mm in diameter), homogeneous, round foci of low signal intensity on T2*weighted MRI or other sequences that are sensitive to susceptibility effects in the areas of cerebellum, brainstem, basal ganglia, white matter, or cortico-subcortical junction. Presence of CMBs was defined as the presence of any CMB (1 point if present).

PSG:

PSG was recorded in a sleep laboratory and readings were averaged over 2 consecutive nights. Recording of PSG started based on the subject's usual sleeping habits and each patient' sleep was recorded for a minimum of 8 hours. The wireless telemetry polysomnography system (manufacturer: Germany SOMNOmedics $₫$ product model: SOMNOscreen plus PSG +, analysis software "DOMINO") was used to monitor sleep. Sleep recordings were scored by a psychologist blinded to the group assignment. All PSG records were staged and scored based on the American Academy of Sleep Medicine Manual[16].PSG measures reported included: sleep onset latency in minutes (SOL, the number of minutes it took for the participant to fall asleep after going to bed),Total sleep time in minutes (TST, total minutes of sleep during the PSG recording); sleep efficiency \% (SE \%; total sleep time divided by time in bed), wake after sleep onset (WASO, the number of minutes being awake after initial sleep onset until last awakening), percentage of each sleep stage (N1, N2, N3 [slow wave sleep; SWS] and RNEM)『apnea-hypopnea index (AHI, \% time oxygen saturation $<90 \%$ ), arousal index ( $\mathrm{Arl}$; total number of arousal divided by the duration of sleep in hours), The cutoff value for higher Arl in CSVD patients was defined as the Arl average score of the participants in control group.

Pittsburgh sleep quality index(PSQI)-Chinese Version: The PSQI is a self-reported questionnaire to assess the quality of sleep over the past month. It contains seven domains including subjective sleep quality, sleep latency, sleep duration, sleep efficiency, and sleep disturbances, use of sleeping pills and daytime dysfunction. Each domain scores from 0 to 3, yielding a global PSQI score ranging from 0 to 21, where a higher score indicates poorer sleep quality [17].

\section{Statistical methods:}

SPSS software package (version 20.0) was used for the statistical analyses. Descriptive statistics were presented as mean \pm standard deviations for continuous normal variables and as numbers or proportions for categorical variables. The student's t test was used for the comparison of sleep parameters between the study and control group. Logistic regression was performed to evaluate the association between sleep 
fragmentation and CSVD imaging markers. Two-sided $P$-values were considered to be statistically significant at $\leq 0.05$.

\section{Results}

Clinical characteristics of participants: 121 consecutive patients with CSVD were screened for recruitment. 37 patients were excluded because they (1) had OSAS $(n=11),(2)$ had other medical conditions that interfere with sleep $(n=2),(3)$ were taking sleep pills $(n=12),(4)$ had major depression $(n=4),(5)$ or refused to participant the study $(n=8)$. A total group of 84 patients and 24 healthy controls were thus enrolled in the study. There were no significant differences in demographic data between the two groups ( $p>0.05$ ), as shown in Table 1. Among the 84 CSVD patients, 63 complained about their sleep quality and 54 patients met the DSM-5 criteria for chronic insomnia.

PSG parameters between the study group and control group: PSG measures showed that participants in study group had significantly lower SE and N-3 ratio compared to control group; while the WASO, N-1 ratio and Arl of study group were significantly higher than control group $(p<0.05)$, which indicated that CSVD patients had more severe objective sleep fragmentation than normal controls, especially during the slow wave sleep, as shown in Table 2.

PSQI measures between the study group and control group: PSQI measures showed that participants in study group had significantly lower SE, worse daytime dysfunction and higher PSQI total score than control group ( $p<0.05)$, which indicated that patients with CSVD had worth subject sleep quality than normal controls, as shown in Table 3.

Imaging markers between the CSVD patients with different level of Arl: among the 84 CSVD patients, 23(27.4\%) patients had LNख25 (29.8\%)had CMB囚42(50\%) had WMH(DWMH Fazekas 2-3 and/or PWMH Fazekas 3$) \varangle 40(47.6 \%)$ had PVS. There was no significant difference in age, sex, education level, BMI and vascular risk factors between patients with higher and lower $\operatorname{Arl}(p>0.05)$. Patients with higher Arl showed significantly more severe PWMH and PVS than those with lower Arl $(p<0.05)$, as shown in Table 4.

The relationship of sleep fragmentation and CSVD imaging markers by ordinal logistic regression: To evaluate the association between sleep fragmentation and CSVD markers of the patients in study group, ordinal logistic regression was performed with PWMH severity and PVS severity as the outcomes; Arl

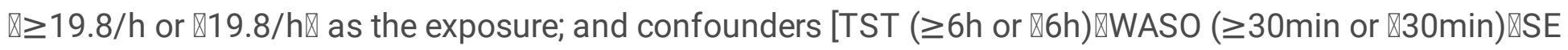
( $\geq 85 \%$ or $₫ 85 \%$ ) and $N-3$ sleep ratio] as the independent variables. The results showed that higher Arl was significantly associated with PWMH severity (OR $1.177,95 \% \mathrm{Cl} 0.170$ to 2.295 ) and PVS severity (OR $1.245,95 \% \mathrm{Cl} 0.485$ to 2.124 ), after adjusting for all the independent variables, which indicated that sleep fragmentation may aggravate pathological process of CSVD, as shown in Table 5.

\section{Discussion}


The first finding of this study was that the proportion of non-breathing-related sleep disorders in CSVD patients was much higher than that of breathing-related sleep disorders. Of the 121 CSVD patients screened, $11(9.1 \%)$ met the OSAS diagnostic criteria, 63(52.1\%) had complaints of insomnia and $54(44.6 \%)$ met the chronic insomnia criteria. This proportion is also much higher than that of the elderly in previous studies [6], and also higher than that of chronic insomnia (29.2\%) in the healthy controls in this study, indicating the high co-morbidity of non-breathing-sleep disorders and CSVD.

The second finding of this study was that the severity of non-breathing-sleep fragmentation in CSVD patients was significantly higher than that in healthy controls. In the elderly, along with the reduced homeostatic sleep pressure and reduced circadian signals, some sleep changes related to aging are often observed: decreased total sleep time, sleep efficiency, and slow-wave sleep; and increased waking after sleep onset [18]. In this study, there was no significant difference in total sleep time between CSVD patients and healthy controls, but the Arl and WASO were significantly higher than those of control group which indicated that the destruction of sleep continuity was more serious in CSVD patients. Accordingly, worse sleep efficiency, lower radio of $\mathrm{N}-3$ and REM sleep, and more impairment of daytime function was observed in CSVD patients. It is well know that CSVD often cause cognitive impairment, this impairment could be aggravated by the decrease of N3 and REM sleep which had been confirmed in our previous research [10].Therefore, the treatment of CSVD cognitive impairment by reducing non breathing-related sleep fragmentation could be a new therapeutic target.

The third finding of this study was that the degree of sleep fragmentation in CSVD patients was positively correlated with the severity of PWMH and PVS. PVS are the extension of subarachnoid spaces that surround the brain's smaller arteries and veins. They are interstitial fluid-filled spaces which act as a glymphatic system and are important in interstitial clearance [19]. Intriguingly, the glymphatic system function mainly during sleep and is largely disengaged during wakefulness. Using real-time twophoton micros copy in live mice, Xie L(2013) found that natural sleep or anesthesia are associated with a $60 \%$ enlargement in the interstitial space, resulting in a significant increase in convective exchange of cerebrospinal fluid with interstitial fluid and a increase rate of $\beta$-amyloid and other metabolities clearance during sleep [20]. Although the glymphatic drainage system in brain may be not the same between humans and mice [21]. Our study support the hypothesis that sleep may promote the metabolite clearance in the human brain through perivascular channels. In this study, it was found that PVS were positively correlated with sleep fragmentation which indicated that destruction of sleep continuity may be associated with inefficiency and blockage of perivascular drainage, potentially resulting in enlargement of the perivascular space.

WMH of presumed vascular origin are very common in elderly and regarded as typical markers of CSVD. Due to limited pathology studies, the underlying pathology of WMH remains ambiguous and is considered rather heterogeneous, the heterogeneity reflecting different disease stages. Possible mechanisms include incomplete infarct, chronic hypoperfusion, immune and inflammatory activation, chronic edema due to increased blood brain barrier permeability [22]. The mechanism by which non breathing-related sleep fragmentation might be linked to WMHs is not clear, but several studies showed that disturbed sleep could 
influence the following factors which might be related to pachologic progress of WMH: decreasing the exchange of cerebrospinal fluid with interstitial fluid and the clearance of metabolities such as $\beta$-amyloid [20], promoting neuroinflammation [23], generating new molecules such as free radicals [24],increasing BBB permeability [25], inducing pulse pressure surges [26], increasing vascular stiffness and altering cerebral perfusion [27]. In the present study, it was found that more severe PWMHs were demonstrated in subjects with a higher Arl, supporting the hypothesis that non breathing-related sleep fragmentation could be related to the pathological mechanism of WMHs.

The strengths of our study are the evaluation of a group of real clinical patients with objective measures of sleep and CSVD markers by magnetic resonance imaging. However, due to the cross-sectional design, we could not draw any causal inference. In conclusion, we found a cross-sectional association between non breathing-related sleep fragmentation and increased severity of PWMH and PVS. Prospective studies are invited to explore the causal relationship of disturbed sleep and the pathological markers of CSVD.

\section{Abbreviations}

CSVD:cerebral small vessel disease

CMBs: cerebral microbleeds

MRI:magnetic resonance imaging

OSAS: obstructive sleep apnea syndrome

PSG: polysomnography

PVS: perivascular space

PSQI: Pittsburgh sleep quality index

SE: sleep efficiency

WASO:wake after sleep onset

WMH: white matter hyperintensity

\section{Declarations}

Ethics approval and consent to participate

The protocol and informed consent documents were approved by the ethics committee of the Third Affiliated Hospital, Sun Yat-sen University. All participants provided written, informed consent. 
Not applicable.

\section{Availability of data and material}

The data sets generated and analyzed during the current study are not available publicly as ethical clearance was not obtained to share data publicly. However,

The data is available from the corresponding author on reasonable request.

\section{Competing interests}

The authors declare that they have no competing interests.

\section{Funding}

This research received no specific grant from any funding agency, commercial or not-for-profit sectors. The authors received no payments for their work.

\section{Authors' contributions}

JHW and XDC conceptualized the study, contributed to the study design, analysed the data and drafted the manuscript. JCL and HYH carried out data collection. LZ and JT contributed to the study design, analysed the data and drafted the manuscript. ZQ lu, as corresponding author, helped to determine the content of the manuscript and guided the overall writing process for this manuscript from start to finish. All authors were responsible for the interpretation of data and critically revised the manuscript. All authors take responsibility for the integrity of the work as a whole, and have given their approval for the final manuscript to be published.

\section{Acknowledgements}

Not applicable

\section{Author information}

${ }^{1}$ Department of Psychiatry, the Third Affiliated Hospital, Sun Yat-sen University, Guangzhou, China. ${ }^{2}$ Department of Neurology, the Third Affiliated Hospital, Sun Yat-sen University, Guangzhou, China. ${ }^{3}$ Department of Rehabilitative Medicine, the First Affiliated Hospital of Clinical Medicine of Guangdong Pharmaceutical University, Guangzhou, China.

\section{References}

1. Cuadrado-Godia E, Dwivedi P, Sharma S, Ois Santiago A, Roquer Gonzalez J, Balcells M, Laird J, Turk M, Suri HS, Nicolaides A, et al. Cerebral Small Vessel Disease: A Review Focusing on Pathophysiology, Biomarkers, and Machine Learning Strategies. J Stroke. 2018; 20(3): 302-20. 
2. Li J, Vitiello MV, Gooneratne NS. Sleep in Normal Aging. Sleep Med Clin. 2018;13(1):1-11.

3. Jehan S, Farag M, Zizi F, Pandi-Perumal SR, Chung A, Truong A, Jean-Louis G, Tello D, McFarlane SI. Obstructive sleep apnea and stroke. Sleep Med Disord. 2018;2 (5):120-5.

4. Song TJ, Park JH, Choi KH, Chang Y, Moon J, Kim JH, Choi Y, Kim YJ, Lee HW. Moderate-to-severe obstructive sleep apnea is associated with cerebral small vessel disease. Sleep Med. 2017; 30:36-42.

5. Feinsilver SH, Hernandez AB. Sleep in the Elderly: Unanswered Questions. Clin Geriatr Med. 2017;33(4):579-596.

6. Patel D, Steinberg J, Patel P. Insomnia in the Elderly: A Review. J Clin Sleep Med. 2018; 14 (6): 101724.

7. Berezuk C, Ramirez J, Gao F, Scott CJ, Huroy M, Swartz RH, Murray BJ, Black SE, Boulos MI. VirchowRobin Spaces: Correlations with Polysomnography-Derived Sleep Parameters. Sleep. 2015;38(6):8538.

8. Del Brutto OH囚 Mera RM, Zambrano M, Lama J, Del Brutto VJ, Castillo PR. Poor sleep quality and silent markers of cerebral small vessel disease: a population-based study in community-dwelling older adults (The Atahualpa Project). Sleep Med. 2015;16(3):428-31.

9. Ramos AR, Dong C, Rundek T, Elkind MS, Boden-Albala B, Sacco RL, Wright CB. Sleep duration is associated with white matter hyperintensity volume in older adults: the Northern Manhattan Study. J Sleep Res. 2014; 23(5):524-30.

10. Wang J, Chen X, Liao J, Zhou L, Liao S, Shan Y, Lu Z, Tao J. The influence of non-breathing-related sleep fragmentation on cognitive function in patients with cerebral small vessel disease. Neuropsychiatr Dis Treat. 2019; 15:1009-14.

11. Wardlaw JM, Smith EE, Biessels GJ, Cordonnier C, Fazekas F, Frayne R, Lindley RI, O’Brien JT, Barkhof F, Benavente OR, et al. Neuroimaging standards for research into small vessel disease and its contribution to ageing and neurodegeneration. Lancet Neurol. 2013; 12(8): 822-38.

12. Fazekas F, Chawluk JB, Alavi A, Hurtig HI, Zimmerman RA. MR signal abnormalities at 1.5 T in Alzheimer's dementia and normal aging. AJR Am J Roentgenol. 1987; 149(2):351-6.

13. American Psychiatric Association. Diagnostic and Statistical Manual of Mental Disorders.5th ed. Washington: American Psychiatric Association; 2013.

14. Staals J, Makin SD, Doubal FN, Dennis MS, Wardlaw JM. Stroke subtype, vascular risk factors, and total MRI brain small-vessel disease burden. Neurology. 2014; 83(14):1228-34.

15. Doubal FN, MacLullich AM, Ferguson KJ, Dennis MS, Wardlaw JM. Enlarged perivascular spaces on MRI are a feature of cerebral small vessel disease. Stroke. 2010; 41(3):450-4.

16. Rosenberg RS, Van Hout S. The American Academy of Sleep Medicine inter-scorer reliability program: sleep stage scoring. J Clin Sleep Med. 2013; 9:81-7.

17. Yu J, Li J, Huang X. The Beijing version of the Montreal Cognitive Assessment as a brief screening tool for mild cognitive impairment: a community-based study.BMC Psychiatry. 2012; $12: 156$. 
18. Suzuki K, Miyamoto M, Hirata K. Sleep disorders in the elderly: Diagnosis and management. J Gen Fam Med. 2017;18(2):61-71.

19. Jessen NA, Munk AS, Lundgaard I, Nedergaard M. The Glymphatic System: A Beginner's Guide. Neurochem Res. 2015; 40(12):2583-99.

20. Xie L, Kang H, Xu Q, Chen MJ, Liao Y, Thiyagarajan M, O'Donnell J, Christensen DJ, Nicholson C, Iliff JJ, et al. Sleep drives metabolite clearance from the adult brain. Science. 2013; 342(6156):373-7.

21. Sokołowski W, Barszcz K, Kupczyńska M, Czubaj N, Skibniewski M, Purzyc H. Lymphatic drainage of cerebrospinal fluid in mammals - are arachnoid granulations the main route of cerebrospinal fluid outflow? Biologia (Bratisl). 2018;73(6):563-8.

22. Shi Y, Wardlaw JM. Update on cerebral small vessel disease: a dynamic whole-braindisease. Stroke Vasc Neurol. 2016;1(3):83-92.

23. Irwin MR. Why sleep is important for health: a psychoneuroimmunology perspective. Annu Rev Psychol. 2015; 66:143-72.

24. Pandey A, Kar SK. Rapid Eye Movement sleep deprivation of rat generates ROS in the hepatocytes and makes them more susceptible to oxidative stress. Sleep Sci. 2018; 11(4):245-53.

25. He J, Hsuchou H, He Y, Kastin AJ, Wang Y, Pan W. Sleep restriction impairs blood-brain barrier function. J Neurosci. 2014; 34(44):14697-706.

26. Lai CT, Chen CY, Kuo TB, Chern CM, Yang CC. Sympathetic Hyperactivity, Sleep Fragmentation, and Wake-Related Blood Pressure Surge During Late-Light Sleep in Spontaneously Hypertensive Rats. Am J Hypertens. 2016; 29(5):590-7.

27. Zhou F, Huang M, Gu L, Hong S, Jiang J, Zeng X, Gong H. Regional cerebral hypoperfusion after acute sleep deprivation: A STROBE-compliant study of arterial spin labeling fMRI. Medicine (Baltimore). 2019; 98(2):e14008.

\section{Tables}

Table 1 Demographic and clinical data 


\begin{tabular}{|l|c|c|c|c|}
\hline Variables & $\begin{array}{c}\text { Study group } \\
(\mathrm{n}=84)\end{array}$ & $\begin{array}{c}\text { Control group } \\
(\mathrm{n}=24)\end{array}$ & $t / \chi^{2}$ & $P$ \\
\hline Age, mean(SD) & $60.1 \pm 8.4$ & $61.8 \pm 10.2$ & $0.136^{\mathrm{a}}$ & 0.728 \\
Male, n(\%) & $47(56.0 \%)$ & $14(58.3 \%)$ & $0.043^{\mathrm{b}}$ & 0.836 \\
Single, n(\%) & $37(44.0 \%)$ & $11(45.8 \%)$ & $0.024^{\mathrm{b}}$ & 0.877 \\
Retirement, n(\%) & $7(8.3 \%)$ & $4(16.7 \%)$ & $1.417^{\mathrm{b}}$ & 0.234 \\
Education $\square 12$ years, $\mathrm{n}(\%)$ & $41(48.8 \%)$ & $15(62.5 \%)$ & $1.401^{\mathrm{b}}$ & 0.236 \\
\hline Insomnia complaints, $\mathrm{n}(\%)$ & $63(75.0 \%)$ & $9(37.5 \%)$ & 11.813 & 0.0010 .002 \\
& $38(51.4 \%)$ & $11(45.8 \%)$ & $0.221^{\mathrm{b}}$ & 0.638 \\
\hline Insomnia, n(\%) & $54(64.3 \%)$ & $7(29.2 \%)$ & 9.366 & \\
\hline
\end{tabular}

${ }^{\mathrm{a}} t$ value, ${ }^{\mathrm{b}} \chi^{2}$ value.

Table 2 Comparisons of PSG measures:

\begin{tabular}{|c|c|c|c|c|c|c|}
\hline Groups & $\mathrm{n}$ & $\mathrm{SOL}, \mathrm{min}$ & TST, min & $\mathrm{SE}, \%$ & WASO, min & AHI, per hour \\
\hline Study group & 84 & $25.3 \pm 14.5$ & $314.8 \pm 88.5$ & $61.5 \pm 10.8$ & $131.5 \pm 51.2$ & $3.4 \pm 1.5$ \\
\hline Control group & 24 & $19.8 \pm 13.8$ & $368.6 \pm 98.2$ & $72.4 \pm 13.4$ & $96.8 \pm 42.1$ & $3.8 \pm 1.2$ \\
\hline$T$ & & 1.054 & 0.796 & 1.985 & 2.325 & 0.124 \\
\hline$P$ & & 0.095 & 0.195 & 0.031 & 0.011 & 0.957 \\
\hline Groups & $\mathrm{n}$ & REM $\square$ \% & NREM-1₫\%ם & NREM-2₫\%ם & NREM-3ロ\%ם & ArI, per hour \\
\hline Study group & 84 & $16.4 \pm 4.7$ & $19.2 \pm 3.4$ & $52.2 \pm 11.4$ & $10.4 \pm 4.5$ & $26.9 \pm 7.4$ \\
\hline Control group & 24 & $19.5 \pm 5.5$ & $12.1 \pm 1.2$ & $53.4 \pm 9.5$ & $13.5 \pm 5.2$ & $19.8 \pm 7.7$ \\
\hline$T$ & & 1.942 & 4.241 & 0.285 & 2.214 & 2.472 \\
\hline$P$ & & 0.045 & 0.000 & 0.657 & 0.039 & 0.021 \\
\hline
\end{tabular}


Abbreviations: SOL, sleep onset latency; TST, total sleep time; SE, sleep efficiency; WASO, wake after sleep onset; REM, rapid eye movement sleep; NREM, non rapid eye movement sleep; AHI, apnea-hypopnea index; ArI, arousal index.

Table 3 Comparisons of PSQI measures

\begin{tabular}{|l|l|l|l|l|l|}
\hline Groups & $\mathrm{n}$ & Total score & Sleep quality & Sleep latency & Sleep time \\
\hline Study group & 84 & $9.3 \pm 5.2$ & $1.5 \pm 1.0$ & $1.3 \pm 1.2$ & $1.7 \pm 1.1$ \\
$T$ & 24 & $5.9 \pm 2.1$ & $0.9 \pm 0.4$ & $0.8 \pm 0.6$ & $1.2 \pm 0.7$ \\
$p$ & & 2.094 & 1.332 & 1.742 & 0.834 \\
\hline Groups & $\mathrm{n}$ & Sleep efficiency, $\%$ & Sleep disturbances & Medication use & Daytime dysfunction \\
\hline Study group & 84 & $65.3 \pm 13.4$ & $1.7 \pm 0.6$ & - & 0.062 \\
Control group & 24 & $76.2 \pm 8.2$ & $1.0 \pm 0.3$ & - & $1.5 \pm 0.8$ \\
$T$ & & 1.893 & 1.022 & - & $1.1 \pm 0.3$ \\
\hline$p$ & & 0.031 & 0.104 & - & 0.030 \\
\hline
\end{tabular}

Table 4 Comparisons of CSVD markers in the patients with different ArI level 


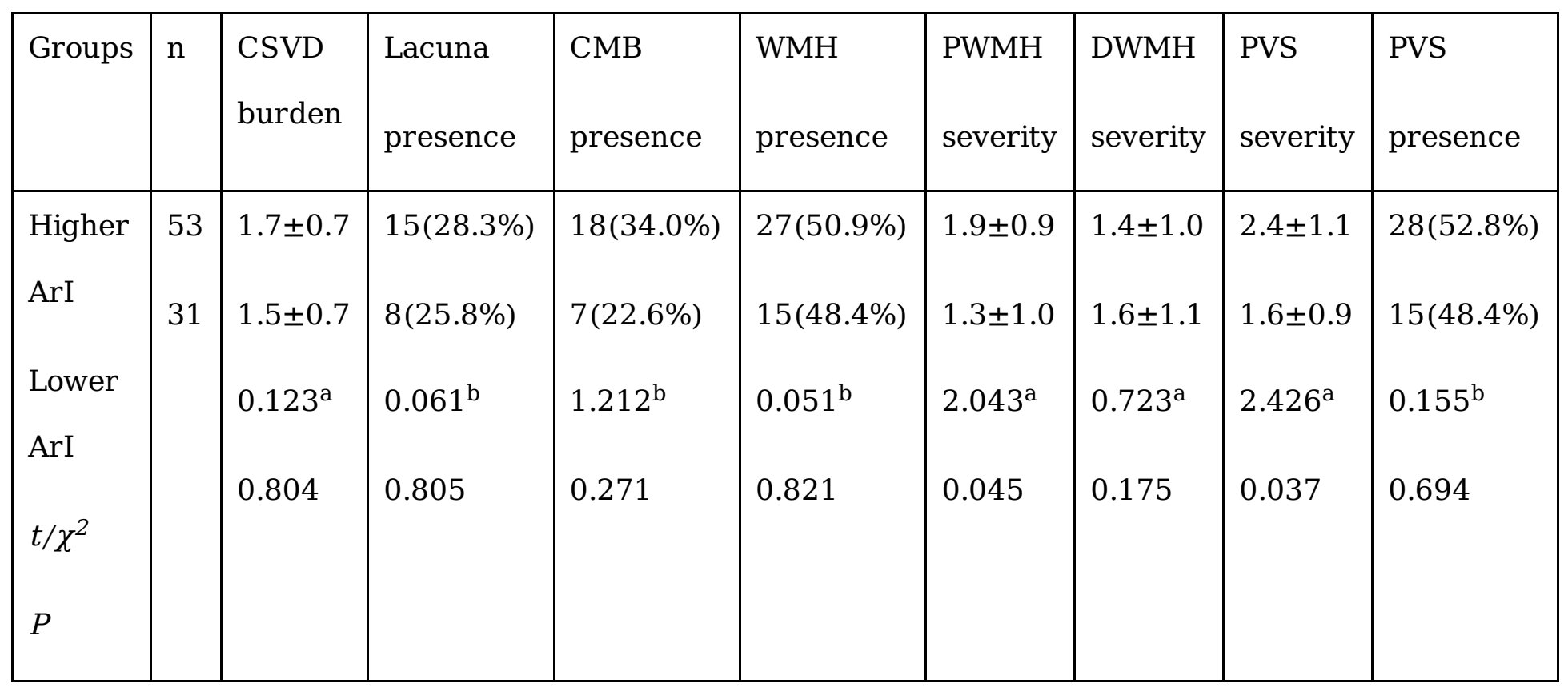

Abbreviations: CSVD, cerebral small vessel disease; CMB, cerebral microbleed; WMH, white matter hyperintensities; PWMH, periventricular WMH; DWMH deep WMH; PVS, perivascular space. ${ }^{\text {a }} t$ value, ${ }^{\mathrm{b}} \chi^{2}$ value.

Table 5 The relationship of sleep parameters and CSVD imaging markers by ordinal logistic regression

\begin{tabular}{|c|c|c|c|c|c|}
\hline Variables & ArI & TST & WASO & SE & $\%, \mathrm{~N}-3$ \\
\hline \multicolumn{6}{|c|}{ PWMH severity } \\
\hline OR & 1.177 & 0.953 & 2.873 & 0.897 & 0.854 \\
\hline $95 \%$ CI & 0.170 to 2.295 & 0.574 to 2.901 & 0.784 to 5.732 & 0.526 to 2.054 & 0.267 to 1.541 \\
\hline$P$ & 0.024 & 0.671 & 0.182 & 0.637 & 0.459 \\
\hline \multicolumn{6}{|c|}{ PVS severity } \\
\hline OR & 2.245 & 1.053 & 1.546 & 1.007 & 1.164 \\
\hline $95 \%$ CI & 1.485 to 4.124 & 0.253 to 2.759 & 0.525 to 2.753 & 0.384 to 1.954 & 0.624 to 2.549 \\
\hline$P$ & 0.019 & 0.533 & 0.337 & 0.723 & 0.239 \\
\hline
\end{tabular}


Abbreviations: PWMH, periventricular white matter hyperintensities; PVS, perivascular space; ArI, arousal index; TST, total sleep time; WASO, wake after sleep onset; SE, sleep efficiency; N-3, non rapid eye movement sleep-stage 3; OR, odds ratio; CI, Confidence interval. 